\title{
Chemical properties of hydroxyapatite deposited through electrophoretic process on different sandblasted samples
}

\author{
Irina Gradinariu ${ }^{1}$, IoAn Stirbu ${ }^{2}$, Cristina Angela Gheorghe ${ }^{1}$, NicAnor Cimpoesu ${ }^{2 *}$, \\ MARICEl AGOP $^{3}$, RAMONA CIMPOESU ${ }^{2}$, CRISTINA POPA ${ }^{1}$ \\ ${ }^{1}$ Dentistry Department, University of Medicine and Pharmacy “Gr. T. Popa”, University Str. 16, 700115, Iaşi, Romania \\ ${ }^{2}$ Faculty of Materials Science and Engineering, "Gh. Asachi” Technical University Iaşi, Romania, \\ D. Mangeron Str. 41, Iaşi, 700050 \\ ${ }^{3}$ Department of Physics, “Gh. Asachi” Technical University, D. Mangeron Str. 67, Iaşi 700050, Romania
}

\begin{abstract}
An implantable material based on titanium (Ti6Al4V) was sandblasted in order to be deposited with a thin film of hydroxyapatite. Two samples of the alloy, in a shape of a bar with $10 \mathrm{~mm}$ diameter and $20 \mathrm{~mm}$ length, were subjected to mechanical treatment. After deposition of the hydroxyapatite through electrophoresis process, the samples were analyzed by scanning electron microscopy. The nature and chemical properties of thin films formed on Ti-based substrate were investigated with electrochemical impedance spectroscopy based on the extremely high polarization resistance of the material. The results revealed the formation of a homogeneous layer on the surface of the metallic substrate. The layer composed of $\mathrm{TiO}_{2}$ and hydroxyapatite provided a high corrosion protection.
\end{abstract}

Keywords: implant; hydroxyapatite; electrophoresis; electrochemical impedance spectroscopy (EIS)

(C) Wroclaw University of Technology.

\section{Introduction}

Surface enhancement is used in metallic biomaterials to improve their mechanical, physical, and chemical properties, such as wear and corrosion resistance, biocompatibility or surface wettability. Also surface modification is usually applied to improve adhesion of new thin films on metallic substrates. In order to improve mechanical retention between two surface areas, one of the surfaces is modified by sandblasting to increase its effective surface area. Mechanically, the procedure of sandblasting is comparable with shot-peening or laser-peening method. The high quality of osteointegration facilitates an accelerated healing process of traumatized bone and ensures high stability and durability of implants. In order to decrease osteo-integration period or to improve the quality of implants, hydroxyapatite (HA) compound can be deposited on a metallic material as a thin

*E-mail: nicanornick@yahoo.com layer [1]. Surface modifications in order to deposit thin films are generally divided into two categories: a concave and a convex texturing. The concave textures of surface can be achieved by either material removal through chemical or electro-chemical action or mechanical indentations (by sandblasting, shot-peening, or laser-peening). Convex texture of a surface can be achieved by depositing different types of particles using one of several physical or chemical deposition methods or by solid-state diffusion bonding [2-4].

In this study, an increase in the effectiveness of surface area has been achieved through sandblasting operation (with different process parameters). Deposition of a HA thin layer was performed by electrophoresis method and then an analysis of structural and chemical properties of the Ti6Al4VHA material was carried out. The electrophoretic deposition of hydroxyapatite was chosen because the film adherence and quality are strongly dependent on the surface roughness. 


\section{Experimental}

Ti6Al4V alloy, in a shape of a bar with $10 \mathrm{~mm}$ diameter and $20 \mathrm{~mm}$ length, obtained from Zirom Giurgiu [5], was used as a support material for the deposition of HA through electrophoresis technique. The samples were sandblasted for 10 and 15 seconds with the use of a Shot Blasting Cabinet model SB974 and classical foundry sand at $7 \times 10^{6} \mathrm{~Pa}$ air pressure, keeping a distance between the gun and the sample of $150 \mathrm{~mm}$.

For the deposition of HA on metallic samples an electrophoresis installation was used and preliminary operations were carried out for chemical activation of the metallic sample by immersion in $\mathrm{NaOH}(10 \mathrm{M})$ solution for 3 hours at a temperature of $60{ }^{\circ} \mathrm{C}$. After activation, the sample was washed in an ultrasound bath with acetone, ethyl alcohol and water for one hour. For the deposition of HA thin film a Consort EV 261 Electrophoretic Power Supply was used to activate the HA particles. The experimental laboratory cell for the deposition is presented in Fig. 1.

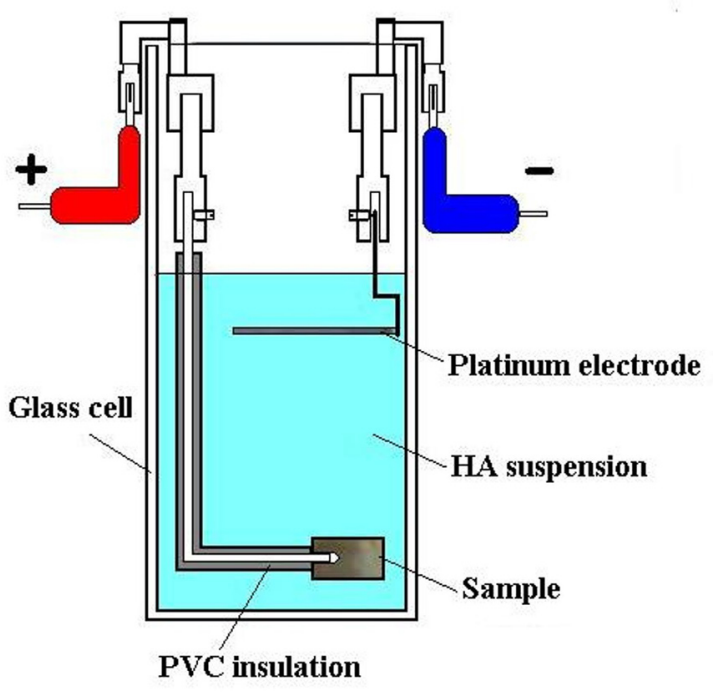

Fig. 1. Schematic view of the deposition cell.

During the process, a voltage of $75 \mathrm{~V}$ was applied between the anode (Ti6Al4V alloy) and cathode (platinum, $\mathrm{S}=6 \mathrm{~cm}^{2}$ ) for 15 minutes using a $20 \mathrm{~mm}$ distance between the electrodes. A suspension of HA powder $(0.61 \mu \mathrm{m}$ mean diameter $)$ in isopropyl alcohol, stabilized with a surfactant Tween 80, was used in the deposition process. The electrolyte was made of $4 \mathrm{~g} \mathrm{HA}$ in $100 \mathrm{ml}$ of isopropyl alcohol $+1 \mathrm{ml}$ of Tween 80 . After the deposition process, the sample was washed with water and dried in a laboratory oven at $110{ }^{\circ} \mathrm{C}$ for 2 hours and calcined at $800^{\circ} \mathrm{C}$ for 2 hours.

The Ti6Al4V alloy surface, after sandblasting and after deposition of HA, was investigated using a scanning electron microscope, model VegaTescan LMH II with SE detector.

The corrosion resistance can be estimated by means of impedance method, also known as EIS. This method is powerful in investigating electrochemical and corrosion systems. This is essentially a steady state technique that is capable of assessing relaxation phenomena, whose relaxation times vary over few orders of magnitude, and permits obtaining an average output within a single complex experiment. The EIS spectra are presented as Bode plots of logarithm of impedance and of phase angle as a function of frequency.

EIS (Electrochemical Impedance Spectroscopy) spectrum was registered in the frequency range of $10^{-2}$ to $10^{5} \mathrm{~Hz}$ at an alternative potential with $10 \mathrm{mV}$ amplitude using a potentiostat PGZ 301 (Radiometer, Copenhagen). Experimental data were converted with EIS file converter software (EISFC150) and then processed with "ZSimWin".

\section{Results}

An appropriate solution to enhance biocompatibility of a metallic implant made from Ti-6Al-4V an alloy widely used in dental and orthopedic applications - is the deposition of a hydroxyapatite (HA) coating on the implant surface [6]. HA coatings were deposited by electrophoresis after the metallic material surface was sandblasted. We analyzed the influence of surface morphology on structural and chemical properties of the thin layer. Even though titan and its alloys are known as hard to be blasted because of their plasticity and low thermal conductivity or chemical reactivity, the material surface can be modified in various ways. Samples 1 and 2 in a form of cylinders of $20 \mathrm{~mm}$ diameter 
and $20 \mathrm{~mm}$ height were sandblasted from $150 \mathrm{~mm}$ distance for 10 and 15 seconds, respectively. In Fig. 2 the surface of Ti6Al4V alloy after sandblasting is presented. In the photo there are also visible sand particles used in the sandblasting process. Even though the procedure was modified by adjusting the sandblasting equipment parameters, in both cases the surfaces displayed irregular profiles with pits and chemical contaminants.

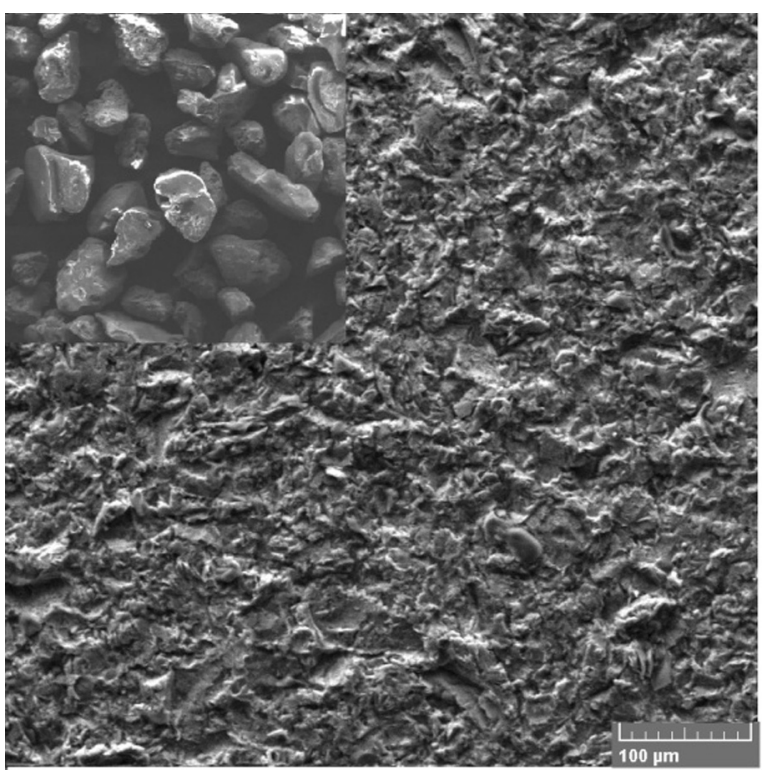

Fig. 2. Surface of Ti6Al4V alloy after sandblasting.

Structural parameters obtained from the SEM images are collected in Table 1.

In order to obtain an estimation of effective area of a surface, modified by sandblasting with sand particles having a $300 \mu \mathrm{m}$ average dimension, a tenth part of the surface, around $30 \mu \mathrm{m}$, should be indented [2]. Using this method we can confirm the expected results through average values of the indents. Average values of $24.9 \mu \mathrm{m}$ and $23.02 \mu \mathrm{m}$, obtained with the use of VegaTescan equipment software for samples 1 and 2 after 10 and 15 second sandblasting, respectively, are presented in Table 1.

An active area of a surface (AS), i.e. an area after mechanical grinding, can be defined relative the original surface A0 using the effective area increment of the surface equal to $(\mathrm{AS}-\mathrm{A} 0) / \mathrm{A} 0 \times 100(\%)$, where $\mathrm{AS}=(\alpha / 360)$ $\times 4 \pi r^{2}$ and $\alpha$ represents the medium effect of sandblasting operation on the surface. Considering the medium value of the sand particles, the average diameter of the indents (Table 1) on each of the original surfaces, the final active surface has increased of almost $95 \%$ in both cases. It can be considered that the active surface has almost doubled for both samples.

Thin HA layers were deposited using the electrophoresis method and the surface was investigated with scanning electron microscopy. The state of the sample surface after the deposition of HA layer is presented in Fig. 3. The structure of the hydroxyapatite layer is homogeneous, in both cases, without micro-cracks and pores.

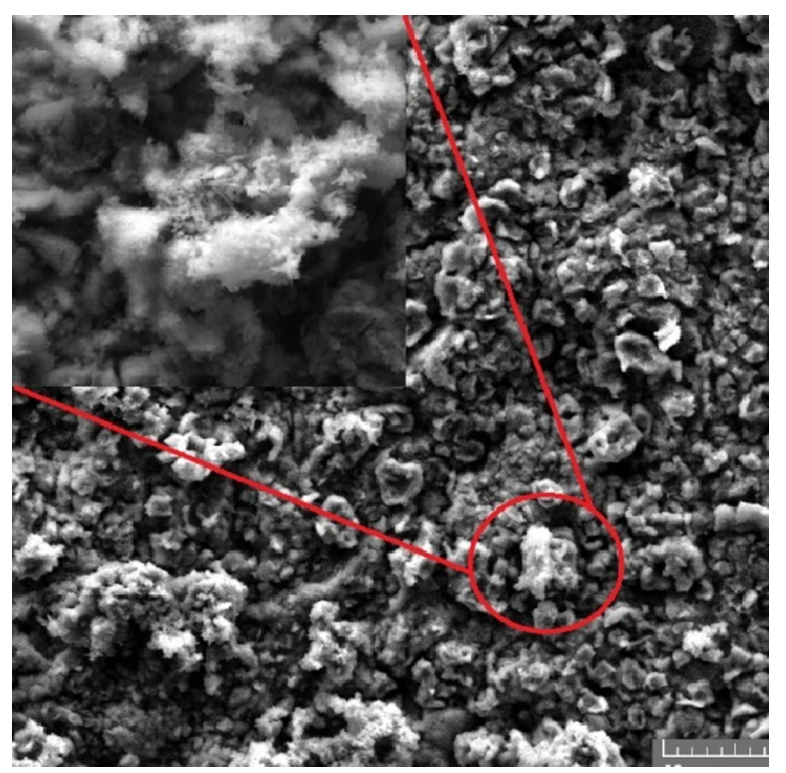

Fig. 3. Morphological aspects of the deposited thin layer.

Samples 1 and 2 were investigated using Electrochemical Impedance Spectroscopy (EIS) in order to analyze the superficial layer structure in a simulated blood serum solution (Simulated Body Fluid - SBF), but five times more concentrated $5 \times \mathrm{SBF}$. The measurements were taken at an open circuit potential in a naturally aerated solution. The schematic representation of this circuit, according to the Boukamp coding [7], is presented in Fig. 4.

The Bode diagrams for samples 1 and 2 are presented in Fig. 5 and 6, respectively. The impedance 
Table 1. Micro-structural parameters of sand-blasted surface.

\begin{tabular}{cccccccc}
\hline \multirow{2}{*}{ Sample } & \multicolumn{3}{c}{ Diameter $(\mu \mathrm{m})$} & \multicolumn{3}{c}{ Standard deviation Depth Coverage degree Final active surface increase } \\
\cline { 2 - 5 } & minimum average maximum & $(\mu \mathrm{m})$ & $(\mu \mathrm{m})$ & $(\%)$ & $(\%)$ \\
\hline \hline 1 & 18.65 & 24.9 & 32.91 & 3.35 & 5.5 & 100 & 95 \\
2 & 13.15 & 23.02 & 38.96 & 6.03 & 6.8 & 100 & 95 \\
\hline
\end{tabular}

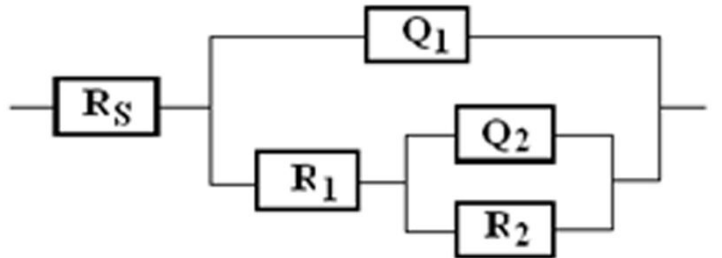

Boukamp: $R(Q(R(Q R)))$

Fig. 4. Equivalent Boukamp circuit.

spectra displayed in Fig. 5 and 6 exhibit two time constants for the tested samples.

They can be divided into two distinct frequency regions: the time variable in the high-frequency part, which arises from uncompensated ohmic resistance due to the electrolytic solution and the impedance resulting from the penetration of the electrolyte through the porous film, and additionally, the low frequency part accounting for the processes taking place at the substrate/electrolyte interface [8]. The Bode plots exhibit a two-step or a two time constant system, indicating a film with two layers, i.e. a porous outer layer and a compact inner layer.

For the interpretation of the electrochemical behavior of a system from EIS spectra, an appropriate physical model of the electrochemical phenomena occurring on the electrodes is necessary. After testing a number of different electrical circuit models, in the analysis of the obtained impedance spectra it was found that the whole set of data for the two samples could be satisfactorily represented by the equivalent circuit (EC) shown in Fig. 4. This is based on the consideration of a two-layer model for the surface film. The EC is similar to that proposed by Pan et al. [9] for titanium immersed in saline solution, by Gonzalez and Mirza-Rosca [10] for titanium alloys in Ringer type solutions, and by Mareci et al. [11] for titanium in artificial saliva. Instead of pure capacitors, the constant phase elements $(\mathrm{Q} 1$ and Q2) were introduced in the fitting procedure to obtain compatibility between the simulated and experimental data. The impedance of CPE is a combination of properties related to both the surfaces and electroactive species, independent of frequency and can be represented by the equation: $Z(\omega)=\frac{1}{Q(j \omega)^{n}}$ where $\mathrm{Q}$ is an adjustable parameter used in the fitting routine. When the value of $n$ is equal to 1 , the $\mathrm{CPE}$ acts as a pure capacitor [12]. The components of the EC are: Rs - ohmic resistance of the electrolyte; R1 - resistance of the outer porous layer; R2 - resistance of the compact inner layer; Q1 constant phase element of the outer porous layer; Q2 - constant phase element of the compact inner layer.

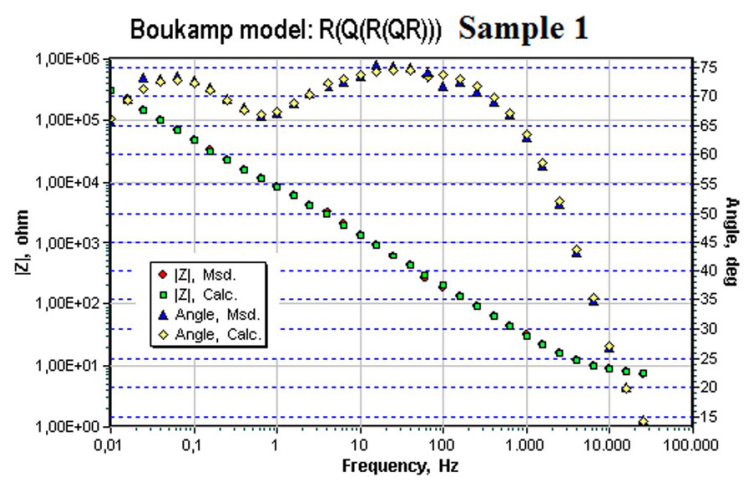

Fig. 5. Bode diagram of sample 1 immersed in human simulated liquid $(5 \times \mathrm{SBF})$.

In Table 2, the main parameters of the proposed EC obtained for the studied samples are presented. The EIS results suggest capacitive behavior (high corrosion resistance) with phase angle close to $90^{\circ}$ and high impedance values (of the order of $10^{6} \mathrm{~cm}^{2}$ ) at medium and low frequencies, which confirm the formation of a highly stable film. According to the proposed model, the passive film 
Table 2. Equivalent circuit elements values describing the superficial layer from the alloy surface after electrophoretic deposition and calcination.

\begin{tabular}{cccccccc}
\hline Sample & $\begin{array}{c}\mathrm{R}_{S} \\
\left(\Omega \cdot \mathrm{cm}^{2}\right)\end{array}$ & $\begin{array}{c}\mathrm{R}_{1} \\
\left(\Omega \cdot \mathrm{cm}^{2}\right)\end{array}$ & $\begin{array}{c}\mathrm{Q}_{1} \\
\left(\mathrm{~S} \cdot \mathrm{s}^{n} \cdot \mathrm{cm}^{-2}\right)\end{array}$ & $\mathrm{n}_{1}$ & $\begin{array}{c}\mathrm{R}_{2} \\
\left(\Omega \cdot \mathrm{cm}^{2}\right)\end{array}$ & $\begin{array}{c}\mathrm{Q}_{2} \\
\left(\mathrm{~S} \cdot \mathrm{s}^{n} \cdot \mathrm{cm}^{-2}\right)\end{array}$ & $\mathrm{n}_{2}$ \\
\hline \hline 1 & 6.69 & $3.80 \times 10^{4}$ & $2.23 \times 10^{-5}$ & 0.843 & $1.60 \times 10^{6}$ & $1.02 \times 10^{-5}$ & 0.908 \\
2 & 5.81 & $3.03 \times 10^{3}$ & $4.88 \times 10^{-5}$ & 0.808 & $5.30 \times 10^{6}$ & $1.62 \times 10^{-4}$ & 0.825 \\
\hline
\end{tabular}

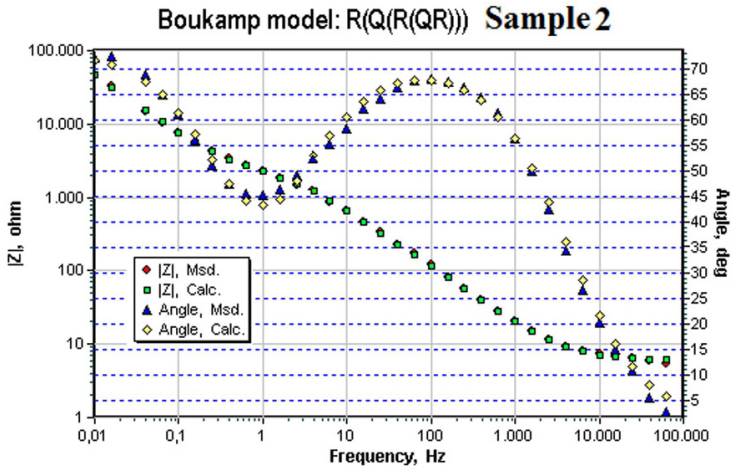

Fig. 6. Bode diagram of sample 2 immersed in human simulated liquid $(5 \times \mathrm{SBF})$.

consists of two layers, the compact inner layer, whose resistance value, R2, is significantly larger than the value associated to the outer porous layer, $\mathrm{R} 1$, as it is shown in Table 2. These results indicate that the protection provided by the passive layer is predominantly due to the compact inner layer.

The value of the fit exponent " $n$ " corresponds to the extent of dispersion and is attributed to surface inhomogeneity. When the value of the exponent $\mathrm{n}_{2}$ is approximately 1 , then CPE 2 can be said to behave similarly to a pure capacitor. The value of the exponent $\mathrm{n}_{1}$ is lower than those of $\mathrm{n}_{2}$; this may be due to higher defectiveness, heterogeneity, and roughness of the outer layer [13].

Polarization resistance $(\mathrm{Rp})$ is represented by the sum of the resistance of the porous oxide layer and the compact oxide layer $(\mathrm{R} 1+\mathrm{R} 2)$ : $\mathrm{Rp}=1.838 \times 10^{6}$ for sample 1 and $\mathrm{Rp}=$ $5.33 \times 10^{6}\left(\Omega \cdot \mathrm{cm}^{2}\right)$.

\section{Conclusions}

The results suggest that the superficial layer on the surface of Ti6Al4V alloy after deposition and calcination is made of a compact layer of $\mathrm{TiO}_{2}$ extremely well adhering to the metal substrate and a less compact, non-homogeneous (semi porous) layer, made of hydroxyapatite deposited through electrophoresis. This ensemble ensures a reliable protection for the alloy surface and at the same time a good biocompatibility.

\section{References}

[1] Miyakawa O., Watanabe K., OKaWa S., Kanatani M., NaKano S., Kobayashi M., Dent. Mater., 15 (1996), 21.

[2] Miyakawa O., Watababe K., OKawa S., Shiokawa N., Kobayashi M., TAMura H., Dent. Mater, 9 (1990), 41.

[3] Wen X., Wang X., Zhang N., Biomed. Mater, 6 (1996), 89.

[4] Piattelli A., Scarano A., Coriglano M., PiatTELLI M., Biomaterials, 17 (1996), 2053.

[5] http://www.zirom.ro.

[6] Zakharov N.A., Polunina I.A., Polunin K.E., Rakitina N.M., KochetKova E.I., SOKOlova N.P., Kalinnikov V.T., Inorg. Mater.+, 40 (2004), 641.

[7] BOUKAMP B.A., Equivalent circuit (EQUIVCRT. PAS), User's manual Dept. Chem. Tech., Univ. of Twente, Netherlands, Raport CT 89/214/128, 1989.

[8] Souto R.M., Alanjali M., Corros. Sci., 42 (2000), 2201.

[9] Pan J., Thierry D., Leygraf C., Electrochim. Acta, 41 (1996), 1143.

[10] Gonzalez J.E.G., Mirza-Rosca J.C., J. Electroanal. Chem., 471 (1999), 109.

[11] Mareci D., Ungureanu G., Aelenei D.M., Mirza Rosca J.C., Mater. Corros., 58 (2007), 848.

[12] PAn J., Leygraf C., Thierry D., Ektessabi A.M., J. Biomed. Mater. Res., 35 (1997), 309.

[13] Souto R.M., Laz M.M., ReIs R.L., Biomaterials, 24 (2003), 4213. 